Send your letters to the Editor,

British Dental Journal

64 Wimpole Street

London

W1G 8YS

E-mailbdj@bda.org

Priority will be given to letters less than 500 words long.

Authors must sign the letter, which

may be edited for reasons of space.

\section{BOTTOMS UP}

Sir, whilst undertaking a survey-based research project as part of an MSc in general dental practice, I offered participants the opportunity to win a case of wine as a gesture of thanks for their cooperation.

The final question in the survey was, therefore, regarding the participants' preference for red or white wine. Of those that responded to the question ( $\mathrm{n}=416$ ), 66.35\% indicated a preference for red wine.

It is reassuring to see that the cardiac health message is being so widely embraced by the members of our profession!

I am grateful to those colleagues who participated in the survey, for their support and encouragement.

T. Fox

Wiltshire

DOI: 10.1038/sj.bdj.2008.466

\section{ENDODONTIC INTERVENTION}

Sir, timing, they say, is everything. The paper by Tickle et al. (BDJ 2008; 204: E8) which reviews the outcome of 174 root canal fillings, appears just one month after the systematic review and meta analysis of 63 outcome studies of endodontic treatments published in the International Endodontic Journal by $\mathrm{Ng}$ et al. (IEJ 2008; 41: 6).

The criteria chosen for standard of treatment by Tickle et al. are those advised by the European Society for Endodontology, whilst the only criteria selected for the identification of the 16 reported failures are tooth loss or reintervention.

I am sure that the authors of both of these papers were represented at the recent British Endodontic Society Spring Scientific Meeting where they would have heard a presentation seeking to define 'evidence-based endodontics'. This requires that the most comprehensive clinical outcome data are placed within the context of clinical experience and of patient preference.

The systematic review uses weighted pooled success rates to analyse the weighted effect of each putative factor that could affect outcomes. It highlights other very important additional prognostic indicators for success besides the single one identified by Tickle et al. (viz the requirement that a root treated tooth is promptly and well restored). The first of these additional factors is recognition of the severity of any pre-operative chronic apical periodontitis. Others are related to the quality of treatment, especially the extension and adequacy of placement of obturating materials, both identified as significant determinants.

My own clinical experience of the treatment or re-treatment of some 25,000 root canals in the last 25 years leads me to believe that the great majority of endodontic interventions do indeed serve to relieve any symptoms of pulpitis and prevent acute apical periodontitis in the short term and that this is most likely to be successful where there is no pre-existing infection. However, clinical experience has also taught me that predictable success in the longer-term prevention and treatment of established chronic apical periodontitis is certainly technique sensitive, not specifically in terms of the details of protocols employed, but generally in terms of the satisfactory attainment of procedural goals; the very same goals identified both by the European Society for Endodontology, and also by analysis of causes of failure emerging from the $\mathrm{Ng}$ et al. systematic review of success rates.
Patient preference is for predictable long-term success.

Those readers who take from Tickle et al. the message that prompt restoration is the golden key to endodontic success, without proper consideration of additional, technique-sensitive determinants, may increase their tally of CPD points, but will also contribute to the expense incurred by patients requiring remedial treatment, and to the profits of the dental litigation industry which counts endodontic failure as one of its principal sources of income.

$$
\begin{array}{r}
\text { A. T. Hyatt } \\
\text { London } \\
\text { DOI: } 10.1038 / \text { sj.bdj.2008.467 }
\end{array}
$$

\section{TOOTH SURVIVAL}

Sir, I write regarding The failure rate of NHS funded molar endodontic treatment delivered in general dental practice by Tickle et al. (BDJ 2008; 204: E8).

This paper has a most misleading title by referring to failure of treatment, when what was actually assessed was tooth survival over an undefined period up to eight years. Out of 176 teeth, 16 were deemed failures: 15 of these were extracted.

Over the last 50 years success/failure of root canal treatment has been defined in terms of healing/non-healing of a periapical radiolucency, which has been a proxy measure for apical periodontitis. This was not assessed in the current study.

Most readers will focus on the conclusions, which, like the title, refer to failure rather than non-survival. No evidenced reasons are given as to why the teeth were lost, but given the non-survival was all in uncrowned teeth, the reason could have been related to tooth fracture. 